essary, and that by such treatment we confine the deformity to its minimum.

Let us take for consideration a case of outward dislocation of the foot complicated with severe injury of the end of the tibia. I.et us suppose that it is at the end of the third or fourth week. No trouble was had in reducing the luxation nor in keeping it in place. There was no manifestation of the latent mischief, except more pain and feverishness than usually attend simple dislocations. But at the end of three or four weeks the surgeon, on removing the dressings, notices that the internal malleolus is more prominent than it should be, that the foot inclines to roll outward, and that the foot is slightly outside the axis of the leg. He may also notice that the ankle is more swollen, tender and painful than usual in ankle dislocations. These features, however, are but slight, and unnoticed by the patient, and indicative of a tendency to rather than actual deformity. The surgeon is apt to attribute them to faulty dress ings, and accordingly he adjusts these to overcome the slight deformity or tendency to deformity. At the next dressing the surgeon learns that he has not succeeded, that the trouble persists, or is even increased, and he again adjusts the dressings to overcome the trouble; but weeks roll round and the deformity becomes more pronounced. If the surgeon be a keen observer he will have learned by this time why the trouble persists; he will have discovered that the lower end of the tibia has enlarged, and that the intermalleolar space has widened. He could not prevent all deformity if he would. No justifiable force would bring the malleoli close enough to clasp as they should the articular process of the astragalus.

After two or three months, during which time special efforts have been made to prevent the threatening deformity, the dressings are finally removed and the patient left to himself and a cane. At this time the deformity is not great, but the tendency to deformity is very great, and the disability of the joint is almost total. The special dressings have held the deformity in check, but all dressings removed and weight borne upon the injured ankle, and the ruin at once becomes apparent even to the patient and his friends. So slight, apparently, was the deformity when the dressing were left off at the usual date in dislocations, that the inexperienced surgeon will incline to believe that rest and splints have done all they can do, and that exercise will complete the cure of the injured joint. Fatal mistake! It is of the utmost importance that rest and splints should be continued. In no other way and by no other means can we keep the deformity and disability within their smallest limits. A steel support reaching from foot to knee, as in talipes valgus-a deformity our case much resembles - should be constantly worn when out of bed. Under no circumstances should the injured limb be subjected to weight unless the ankle is supported by an efficient splint, and this adequate support must be kept up until the tenderness, pain, and weakness have disappeared, which will certainly not be short of a year, and it may take two years.

But the inexperienced surgeon of our hypothetical case, at the end of two or three months, surrenders his patient to a cane and exercise. The patient begins timidly to bear some weight on the injured ankle. Even slight weight brings discomfort, but being a determined fellow, and being told that exercise will strengthen the foot, he bravely walks on the foot with the aid of his stick; but it is a limping, painful gait, growing more painful every day until the disability of the ankle is complete. In alarm he sends for his surgeon to "break the leg over." The removal of the sustaining splints and the bearing of weight on the ankle result in displacing a foot that was not tied in its place by internal lateral ligaments. What was only a slight displacement, but a great tendency to displacement, has by injudicious management become an ugly deformity and a painful disability.

\section{CLINICAL NOTES ON THE TREATMENT OF PUL- MONARY DISEASES BY SATURATED VAPORS AT A HIGH TEMPERATURE.}

Read before the Mississippi Valley Medical Association, at Crab Orchard, $K y$., Fuly 14,1887 .

BY G. W. MCCASKEY, A.M., M.D.,

PROFESSOR OF DISEASES OF THE CHEST AND THROAT, FOKT WAYNE COLLEGE OF MEDICINE, FBLLOW OF THE AMERICAN ACADEMY OF MEDICINB, ETC.

The use of saturated vapors at a high temperature in the treatment of pulmonary diseases was made the subject of a paper presented by the author to the Section on Practice of Medicine, etc., at the late meeting of the American Medical Association, in Chicago. To that paper, which is published in The Jounnal of July 23 , I must refer you for a full discussion of the principles upon which the treatment depends. The importance of the subject of topical medication of the lungs can scarcely be overestimated. The meagre successes which have heretofore crowned our efforts, instead of repressing the true spirit of scientific investigation, can only serve to spur it on to renewed attempts. The accessibility of the respiratory organs, although not so easy as at first thought might appear, is yet sufficient to fully justify the therapeutic methods in question.

The advantages of saturated vapors at $140^{\circ}$ to $160^{\circ} \mathrm{F}$. over other forms of medication, seem to me sufficiently apparent in the light of acknowledged laws and a considerable clinical experience. It is for the purpose of "calling attention to this clinical experience, which was necessarily crowded out by the purely scientific discussion in the paper above referred to, that this subject is again presented. It is not my intention to offer a record of cases from my note-book, but rather to speak of the clinical methods and results in a more general manner. A little consideration must first be given to the technique of the special form of apparatus which my experience and experimental investigations have led me to adopt.

The apparatus, which I herewith present to you, is capable of holding at a complete degree of vapor saturation one cubic foot of air. This quantity will more than supply the respiratory volume for one 
minute, however deep the respirations may be; and the same quantity can be heated and saturated in about that length of time, so that a uniform condition can be maintained. The only appliances required in addition to the vaporizer are a spray tube and a compressed air cylinder. When the air in the vaporizer has been heated to the desired temperature, or while it is being heated, a dense spray is thrown in. This spray, entering the heated atmosphere, is instantly vaporized, and this whether it holds in solution volatile or non-volatile agents. So far as I know this is the first effort at a systematic and scientific application of saturated vapor to the respiratory organs by a mechanism which is constructed with a view to meet the physical conditions present. The dry and wet bulb thermometers furnish an accurate means of estimating the quantity of medicine held in solution in the vapor, even to the minute fraction of a grain; while the reduction of temperature which it undergoes in passing into the respiratory organs, in so far as that can be estimated, furnishes an accurate measure of the quantity deposited.

As I have pointed out in the paper already referred to, air saturated at $158^{\circ} \mathrm{F}$. is more than 30 per cent vapor.; and assuming that the respiratory organs would cool it down to $1 \mathrm{I} 3^{\circ} \mathrm{F}$., it would deposit 69 per cent. of its contained vapor. These figures are not based upon hypotheses, but are simply mathematical statements deduced from incontrovertible and fully accepted physical laws. The condition of this medicine when deposited upon the mucous lining, is that of a liquid holding in solution the medicine contained in the original menstruum, and of precisely the same strength. There are no problematical factors or additions, as in the case of the steam atomizer, where the steam mixes in uncertain proportions with the pulverized solution. The secretion with which the mucous lining is constantly bathed is of course an obstacle to the local effects of the medicine. But this objection applies to all other methods of topical medication; and with even greater force than to this. If the deposit of vapor is kept up sufficiently long, the mucus must be correspondingly diluted and thinned, and the structures beneath rendered more accessible.

Whatever hypothetical objections there may be, however, to the local action of agents applied to the respiratory lining, they are completely refuted by the results of clinical observations. The rapid absorp tion of vaporized anæsthetics, along with many other facts, clearly proves that the mucus which bathes the bronchial lining is not such an impassable barrier as some would have us believe. Besides all this, let us not forget that the mucous glands, and consequently the mucous secretions, are entirely absent, not only from the alveoli and air cells, but also from the bronchial tubes of small size. ${ }^{1}$ Consequently any agents carried into the small bronchial tubes finds no tenacious mucus, or any other kind of mucus to guard the structures beneath.

The sensations of the patients, in so far as these can be trusted, are proof of the deep penetration of

\footnotetext{
3 Landois, vol. x, p. 220; Frey's Histology, p. 450.
}

the vapors, if any proof were needed. A feeling of warmth pervades the entire chest; its intensity depending upon the temperature of the vapor, together with the depth and number of consecutive respirations. Occasionally considerable coughing is produced. But in the large majority of patients toleration is soon established. Also in some patients a sense of suffocation is produced by the vapors of higher temperature, which can not be overcome. One or two patients could not respire above $130^{\circ}$ or $135^{\circ} \mathrm{F}$.

The quantity of spray required for each treatment is quite large. About 3 iss-ij of solution will be converted into spray and vaporized for each treatment of ten minutes' duration. I use an air-pressure of about 15 lbs., and keep the spray going diring about one-half or two-thirds of the time of the treatment, relying, of course, upon a comparison of the thermometric scales as to the completeness of saturation. So long as the wet bulb thermometer stands as high as the dry, no additional spray is required, as it would simply fall to the bottom, the air being already charged to the fullest extent possible.

My experience has been principally in the treatment of phthisis and bronchitis, in both of which diseases I have used preumatic treatment in combination with the saturated vapors. My opportunities for comparison extend over a period of about fourteen months, during which I have treated about sixty cases. Increased intra-pulmonary pressure has been used in nearly all the cases, and a compar ison can only be made under similar conditions be. tween the use of the vaporizer, and simple spray or the steam atomizer. As regards sprays at ordinary temperatures, I have no besitation in pronouncing them comparatively inefficient in lesions of the deep respiratory passages. I do this from both theoretical considerations and practical observations; and with a full recognition of their value, and, indeed, their superiority in laryngeal and tracheal inflammation. Aside from their failure to adequately reach even the deep bronchial structure, sprays have a very objectionable feature in the treatment of lesions located here, in their profuse deposit in the mouth and throat where they are not needed.

The steam atomizer fills the indications much more completely, but lacks in the very essential elements of precision, and complete control, both as regards strength of medicine used, and temperature of application. The steam atomizer, although much superior to the ordinary spray, can not be made effectually to do the work of the "vaporizer."

I have lately been using a I per cent. solution of carbolic acid in the treatment of phthisis and the purulent stage of bronchitis. Although it is not open to demonstration, and therefore must be asserted guardedly, yet I am very fully convinced that the vapor, aided by the mechanical effects of increased air-pressure, does penetrate, and is deposited upon, the walls of patulous tubercular cavities. The modification of secretions and of general symptoms can scarcely be explained upon any other hypothesis; besides which the physical laws and conditions are such as to fully justify this explanation. 
A variety of other agents has been used, especially $\mathrm{HgCl}_{2}$, I-1000 to I-2000; but I think the best re sults have been obtained by the use of carbolic acid.

In the treatment of laryngeal affections I do not think that saturated vapor offers any advantages over the common spray or steam atomizer. The quantity of medicine deposited in the laryn $\mathrm{x}$ is probably not so large as it would be with the spray or steam atomizer; but I think that theoretical consid. erations and practical results justify the conclusion that for the deep portions of the respiratory tract it is greatly superior to these or any other method with which I am acquainted.

With the combined effect of pneumatic treatment and saturated vapors, I have seen expectoration diminish and the pathogenic organisms of phthisis grow less and disappear. I do not mean to say that this has been the rule; for with this or any other treatment at present known the arrest of advanced phthisis must be the exception, and a fatal termination the ruie. But I think that a method of treatment which appeals so strongly to our judgment through our knowledge of physical laws, is at least entitled to careful and thoughtful consideration.

In many cases of intractable bronchitis the results have been so signal as to remove all doubts, in my own mind, regarding the superiority of this method. These patients have been treated almost to the exclusion of the whole list of nauseant expectorants; the principle effect of which, in my opinion, is to disturb digestion and destroy appetite. There is an a priori absurdity in mixing a teaspoonful of an expectorant mixture with some fifteen or twenty pounds of blood, as is done after absorption, and then ex. pect a tangible result upon an extensive inflammatory process involving almost the entire bronchial lining. The dose, when it reaches the lungs through the laboratory of the portal circulation, has reached so many "dilutions" that it ought to satisfy the views of Hahnemann's most ardent disciples. While very far from denying internal medication a legitimate place in the therapeutics of pulmonary disease, yet I would make it as subordinate as it is acknowledged to be in the treatment of other inflammations as accessibly located as these. When dealing with a cystitis or pharyngitis, or even a gastritis, we do not rely upon the introduction of medicinal agents into the circulation, from thence to reach the seat of morbid action by a circuitous route, and in infinites. imal dilution. But we apply our remedial agents to the diseased focus by means of the catheter, the gar. gle or probang: and the siphon tube or stomach pump. Constitutional measures are not neglected, but they are not relied upon as the principal method of treatment.

The future, nay I believe the present therapeutics of certain pulmonary diseases must rest upon this basis of local therapeutics, backed up by such constitutional measures as will improve nutritive processes in general, and consequently those of the lungs in particular.

Ft. Wayne, Ind.
CEREBRAL SOFTENING; WITH REPORT OF A CASE.

\author{
BY C. N. BLOUNT, M.D., \\ OF HAGERSTOWN, IND.
}

Daniel N., aged $5^{2}$, carpenter, twenty-five years before his last sickness was suddenly attacked with a severe convulsion. There were no premonitory symptoms, excepting that for a little while before he stammered in attempting to talk. He had about twenty convulsions in all. The paroxysms were very severe, occurring sometimes once or twice a day, and then a day or two would intervene before their return, and thus continuing until they entirely ceased in a short time. There was no tendency to a return of the convulsions until his last sickness. There was no paralysis except aphasia for a short time. He was an invalid, however, for about a year, when he was sufficiently restored to resume his trade, which he continued during the intervening years till his last sickness. He did not seem in any way different from his former self excepting a loss of memory. His wife thinks that his memory was more defective in every way than before the attack, but especially in remembering where he had placed anything he had been using.

There was a small tumor on the back of his head, which gradually grew during these years to a diameter of three inches. He occasionally complained of severe pain in it. It was there long before the above-mentioned attack.

In April, I 885, the tumor was removed by the knife. After the operation he continued at work until October, when he was compelled to give up. His wife thinks, however, that he was never well after the operation. He was peevish, fault-finding and very irritable, becoming very angry at little things. He now frequently complained of headache, which daily increased. He would sometimes say that the old pain was in the back of his head, and would think that the tumor was returning. For a month or more before he took to his bed, he was very much indisposed. He acted strangely, slept badly, was feverish at night, complained of numbness of limbs, was costive. There was no nausea, and his appetite was good all the time. He persisted in being at work up to October, i 885 , when he had to take his bed.

He occasionally had slight convulsions during October, November and December. Sometimes during the summer he would forget words, but this was now much more noticeable. Most of the time during his sickness he had hemiopia; a few times he had diplopia. He spoke of this last trouble only on two or three occasions, but the hemiopia was rather persistent. There was a gradual failure of both physical and mental forces until he was a complete physical wreck and demented, taking no interest in anything. During the latter part of his sickness there was partial paralysis of the right side, more marked in leg than arm. There was also paralysis of the tongue and muscles of the pharynx. This was very distressing during the last two months of sickness. He could scarcely speak or swallow. He became very much emaciated, and dièd in April, 1885 , about one year after the removal of the tumor. 